\title{
IDEAL TOOTH BRUSHING
}

\author{
WILLIAM J. CHARTERS
}

Des Moines, Iorwa

CONTENTS

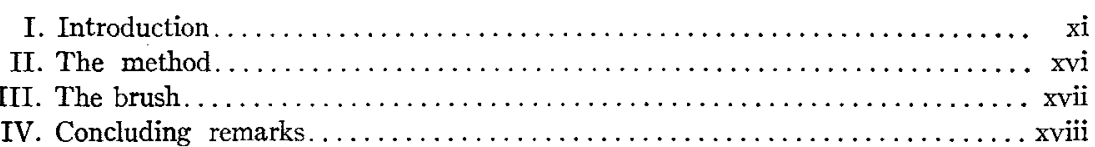

\section{INTRODUCTION}

Of all the diseases by which mankind is afflicted, the most prevalent are diseases of the teeth and gums. Pathological conditions have always been present in the mouth, but unlike many other diseases, which have diminished in severity in modern times, abnormal mouth conditions have been growing steadily worse. I believe that more good can be accomplished by the dentist, in our day at least, in teaching patients how correctly to clean the oral cavity, than by dietitians in trying to regulate the diet so that tooth brushes will not be considered a necessity. One of these two systems must be perfected, "with results," before the people will be relieved of many diseases from which humanity is suffering.

It is not my intention to discourage the teaching of the use of proper diet, but it is a much easier task to teach a correct method of brushing the teeth than it is to wean the average person from his taste for jam and French pastry. Therefore, let the dental profession take another step forward and band together in the great cause of teaching real preventive dentistry, so that patients will realize the necessity for dental examinations at intervals of six months, and that, by faithfully brushing the teeth in the proper manner, their mouths will be kept in practically perfect condition.

It has been said that "a clean tooth never decays." This sentence was cut too short--there should have been added: "and stimulated gums never have pyorrhea." People without dental decay are led to believe, after hearing the phrase, "a clean tooth never decays," that they are free from

1 Read at a meeting of the American Academy of Periodontology, in New York, May 10, 1921. 
mouth ills, when, if the truth were really known, they may be in a more serious condition than they would be if dental decay were their only oral abnormality. One of the most treacherous characteristics of pyorrhea is the fact that, in 95 per cent or more of the cases, no pain is present, with the result that first examinations often reveal unsuspectedly serious conditions.

About eight years ago I began to develop a method of teaching my patients how to keep their mouths cleaner. This effort was prompted by the fact that my patients would return in six months or a year with mouths that were as unhealthy as when they were first examined, and my work seemed to be in vain. I procured an artificial set of teeth, a tooth brush, and some vaseline that I colored black. Then I plastered the inter-proximal spaces with the colored vaseline. By using the up-and-down brushing motion, I found that it was impossible to remove the paste from between the teeth. Then I tried another brushing method in vogue, the rotary method, with results that were not as good as those of the first test. Finally, it occurred to me that, to clean a surface, the cleansing instrument must come into contact with that surface. Taking my brush I pushed the bristles between the teeth and then gave the bristles a slight rotary or vibratory motion. Very soon I was convinced that I was obtaining results.

In this last test I learned I could stimulate the gums with the sides of the bristles. I also learned that the brush does not effectively remove food film from the mouth without thorough rinsing. The next, and most important step, was to try the method thoroughly on my own mouth. When I pushed the bristles in between my teeth, I was immediately convinced that my brush had touched parts of my gums that had never felt a bristle before.

While brushing is effective as a cure, its greatest merit is its utility in real prevention. Our patients will have greater respect for and confidence in our work after we teach them that most all tooth surfaces can be cleaned with a tooth brush, and that most of the brushing nowadays only hits the high spots, where decay seldom starts. The attention of the patient should be called to the fact that there are five surfaces to every tooth that should be brushed, namely, mesial, distal, occlusal, buccal, and lingual. The average person brushes only two surfaces, namely, buccal and lingual, and firmly believes that he has done a perfect job, when in reality he has not even started. I believe most people have learned incorrectly how to brush their teeth through instruction from the platform and in tooth brush drills, or by reading tooth-brush or tooth-paste advertisements. 


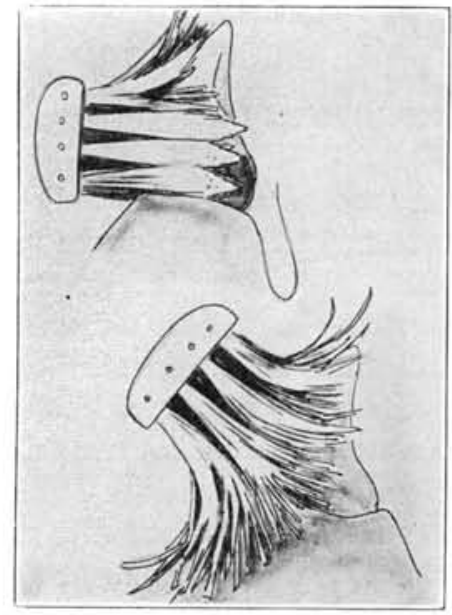

Fig. 1.

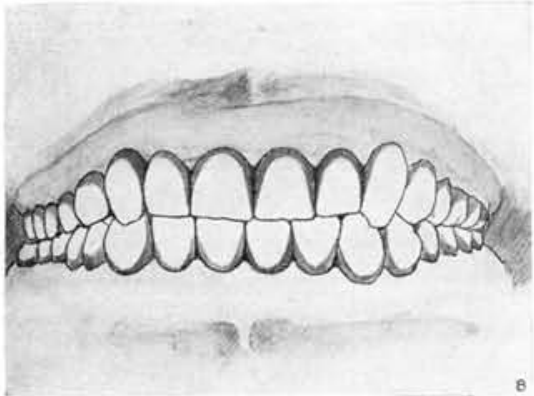

Fig. 8.

Fig. 1. Upper. $-\Lambda$ drawing that shows that the approximal surfaces may be cleansed when the bristles of the brush are properly placed. Lower.-A drawing that indicates the difficulties the beginner experiences in his efforts properly to place the bristles of the brush between the teeth; a difficulty that can be readily overcome by practice.

Fig. 8. A Drawing That Shows the Conditions Remaining After Completion of ANy of the Old Methods of BRUShing the TeEth

The dark areas around the gingiva represent undisturbed portions of food debris, mucin film, etc.

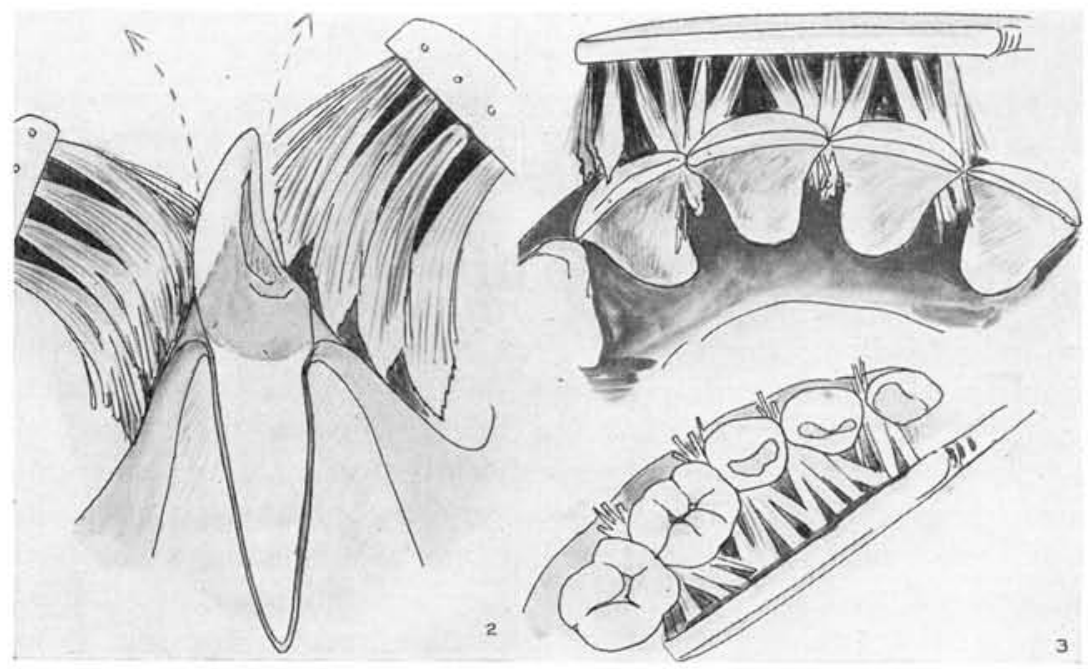

Fig. 2.

Fig. 3.

Fig. 2. A Drawing that Shows the Old Up-and-Down and Rotary Methods of BRUSHING THE TEETH

Note the existence of uncleansed areas between the contact points and the gums.

Fig. 3. A Drawing That Shows the Buccal and Lingual Positions of the Bristles, When the Teeth are Brushed by the Ideal Method 
Removing sensitive conditions from exposed roots is easily accomplished by the method of brushing I have suggested-and this is one of the very difficult conditions we have to contend with. The most sensitive tooth surfaces can be desensitized in a few minutes; and in an hour's time, the same sensitive condition may return and continue to return after each brushing for a few days. In three or four weeks' time, however, most of the bacteria will have been eliminated, the resistance of the remaining bacteria is lowered, the fermentation acid is less abundant, and the dentine, which at the start was hypersensitive, is insensitive to the touch of a steel instrument. I have cured many such cases with no other instrument than a tooth brush.

I have had cases of muscular rheumatism that were cured by brushing only, and there are cases on record where eczema has been made to clear up, and many cases of initial pyorrhea have been eliminated. That tired and "no-pep" feeling is the most easily handled of all other conditions. I find cases can be treated effectively in two weeks.

Several years ago Madam Montessori originated a method whereby small children could be taught to feed themselves, sew, and in fact do everything mechanically correct. She made a wonderful success of this method, and many of her theories are being used in educating the small child today. If children can be taught to do these things correctly, they can be taught to brush their teeth correctly. I ask you to give this method a fair trial, using normal children for your subjects. You will find that in most cases they respond and learn the method much more readily than adults.

Some will say, perhaps, that they are afraid this method will cause undue recession of the gums. In answer to this I want to say that I have examined the mouths of hundreds of children and have never yet seen such a case following the use of the method. You cannot drive the tissue from the palms of your hands, or the soles of your feet. Nature cares for this and builds up a resistance in the tissue to withstand any pressure applied.

The rotary method is half way between the horizontal motion and the up-and-down motion, and to your surprise I make the statement that the horizontal motion is more effective than the up-and-down motion. Tests on artificial teeth have proved this. In orthodontic cases, which are in process of correction, a mechanical appliance is used. Can you imagine it possible to stimulate the gum margin, and clean the teeth under such appliances, with the up-and-down or the rotary method of brushing?

Children undergoing orthodontic treatment have a fine opportunity to learn the home care of the teeth, for they make many visits to dental offices before their cases are completed. If the method I am presenting 


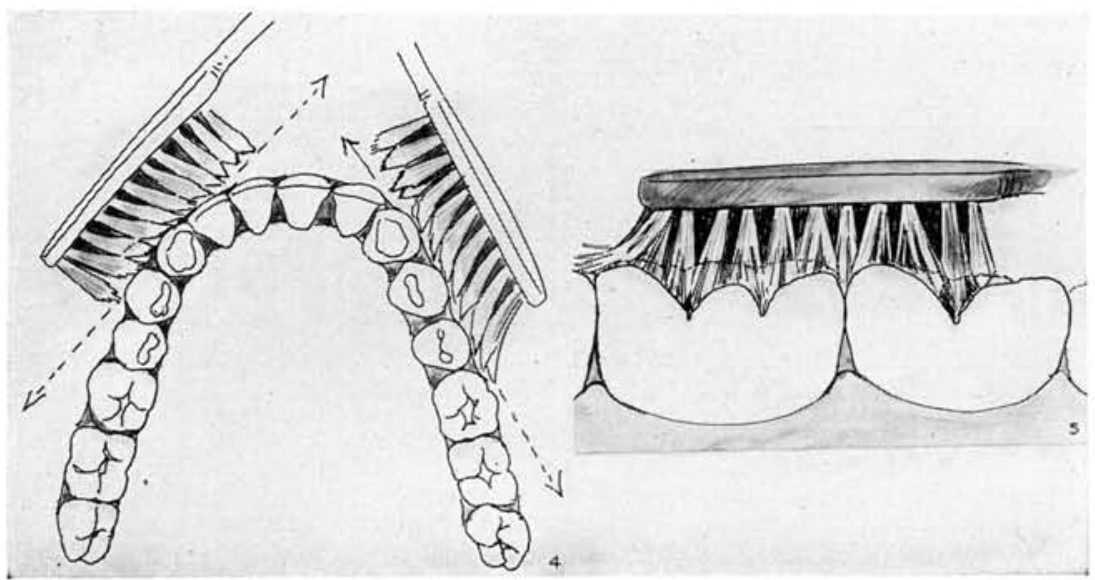

Fig. 4.

FIG. 5.

Fig. 4. A Drawing That Shows an Old Method of Brushing the Tefth

The bristles merely cleanse the most prominent portions of the teeth, which normally are clean areas.

Fig. 5. A Drawing That Shows a Feature of the Ideal Method of Brushing THE TEETH

The bristles are pushed into, and moved about in, the fissures and sulci

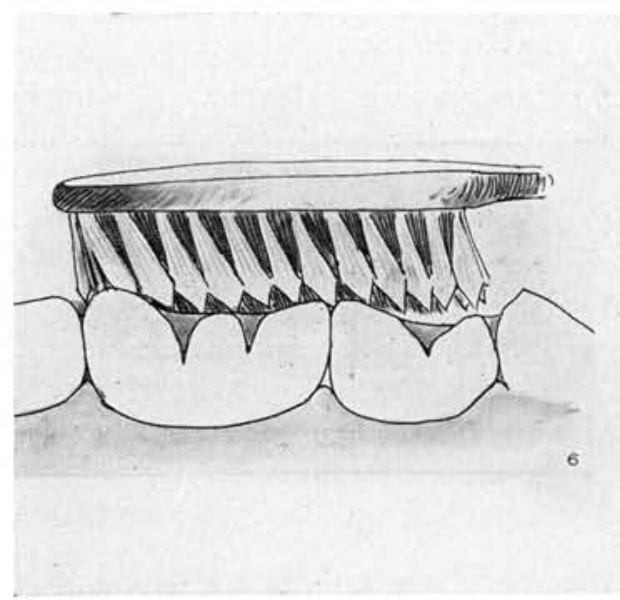

FIG. 6.

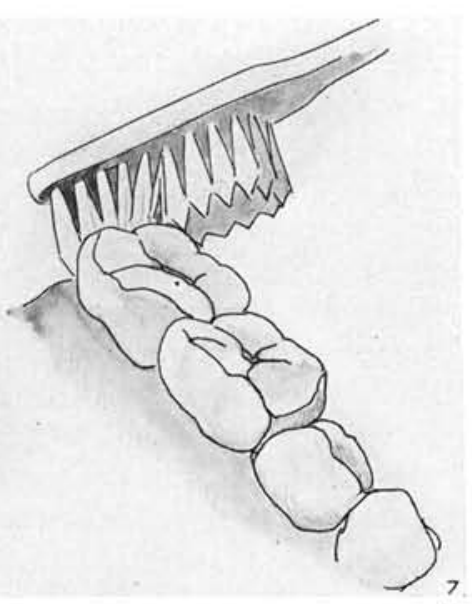

FIG. 7 .

Fig. 6. A Drawing That Shows an Incorrect Method of Brushing Occlusal SURFACES

The bristles touch only the high points and glide over the fissures and sulci

Fig. 7. A Drawing That Shows the Correct Method of Placing the Brush in

Order to Reach the Seemingly Inaccessible Distal Surfaces of the Molars 
to you is ideal for such mouths, it certainly must be ideal for any other condition, either normal or sub-normal.

Every local patient who presents his or her case for pyorrhea treatment is required to take a two-weeks course in brushing before curetting is started. A urine analysis is also made at the first sitting. If trouble is located, the patient is informed of the condition and is told to visit the family physician. The patient is also instructed to bring two tooth brushes, which are re-shaped. One is returned to the patient for home work, and the other is placed in a large test tube, which is labeled and dated, and is kept in the office for brushing and demonstrating. Appointments are made with the patients for every day, if possible, or at least every other day. The nurse who gives the lessons consumes about ten minutes at a brushing, and ten minutes in instructing the patients how to manipulate the brush. Disclosing solution is used to show the patients the surfaces they may be missing.

\section{THE METHOD}

Place the brush at right angles to the long axis of the teeth, the point of the bristles in contact with the surfaces. Then gently force the bristles between the teeth, being careful not to pierce the gum, that is, do not allow the points of the bristles to rest on the gums. With the bristles between the teeth, exert as much pressure as possible, giving the brush several slight rotary or vibratory movements, causing the sides of the bristles to come in contact with the gum margin, producing an ideal massage. Be careful not to make this movement sufficiently extensive to remove the bristles from the spaces between the teeth. After making three or four small circles, remove and then replace. Make three or four applications in the same place, so that the points of the bristles will strike the labial, buccal and lingual surfaces; then slide them into the inter-proximal spaces. Move the distance of one embrasure and repeat the same process, holding the sides of the bristles firmly on the gum margin. The V-shape space between the teeth assists in crowding the sides of the bristles against the gingiva. However, when teeth are missing one must depend on his sense of touch to maintain the proper pressure, with the sides of the bristles on the gum margin.

Enter every embrasure from both the outer and inner surfaces, on both the upper and lower teeth. Most of the third molars, which, because of the close proximity of the ramus of the jaw and the soft tissues in that region, cannot be approached at right angles, must receive more careful attention. The brush should be tipped so that the bristle ends reach the 
outer and inner sides. Third molars in proper alignment and occlusion can be saved as long as any other teeth, if they are kept equally as clean. Last, but very important, do not brush the chewing, occlusal, or grinding surfaces with a sliding or sweeping motion, but place the brush vertically upon this surface, then gently, with a slight rotary movement, force the bristles into the pits and fissures. Remove the brush and repeat the process until the entire chewing or biting surface has been brushed.

After the teeth have been thoroughly brushed according to the foregoing method, the next important point for consideration is proper rinsing of the mouth. The brush can remove very little debris from the mouth. The "food film" and saliva comprise a liquid that fills the fissures and embrasures. It is very important that the debris be washed out of the mouth. After taking a mouth full of luke warm water, occlude the teeth and lips, and force the water back and forth through the inter-proximal spaces frequently with as much pressure as possible. Use an abundance of water for this purpose. It is better to wash the bacteria out of the mouth than to try to destroy them in the mouth with antiseptics.

\section{THE BRUSH}

The brush should be seven-eighths of an inch from heel to toe, and fivesixteenths of an inch in width; the bristles, one-half an inch in length. The tufts should be separated from each other by a space about the width of the tuft. The top of the tufts should be saw-shaped. The handle of the brush ought to be 7.5 inches in length, rigid, and preferably of bone. The fine Russian bristle, by far the best, cannot be procured at present. Therefore, we are forced to go to the other extreme and use a coarse bristle, for this method, I must admit, is hard on bristles, especially in the hands of the beginner.

One member of each of many families using this method of brushing their teeth has been instructed in my office, or in another dental office, and in return has been an efficient teacher in his home.

I think you will agree with me when I say that practical demonstration in the mouths of dentists is the best way to make this method clear. Real experience is the best teacher and makes most lasting impressions. Several lessons will be necessary to teach the proper use of this method. After the first lesson I am sure you will be convinced and brought to a realization of the value of this method. 
xviii

\section{CONCLUDING REMARKS}

After a few years of uphill work I organized the Des Moines ToothBrush Clinic, which has given demonstrations at three national dental conventions, and at several state meetings. If each district dental society would organize a tooth-brush unit, we should be able to reach all the dentists in a comparatively short time. Would it not be worth the effort?

I am going to show here for your consideration some $x$-ray slides demonstrating a phase of work in which I am intensely interested, that of deceitful pyorrhea. In diagnosing mouth conditions, by exploration, I have often found minute pockets extending deeply into the process between the teeth, when the $\mathrm{x}$-ray failed to reveal them. I believe these conditions could have been prevented if the gum and process had been properly stimulated by the ideal method of brushing that I advocate.

This method is not an experiment. I have been teaching it to my patients for years, and there is no other one thing in my practice that has given me such lasting and genuine satisfaction. The pleasure of having my patients come back, year after year, with clean teeth and beautiful, pink, hard gums, is worth more to me than money. I earnestly advise that you make the teaching of this method to your patients a part of the routine of your practice. 